\title{
To MUF or not to MUF, that is the question
}

\author{
Jordan Bloom $^{1}$ and Gus Vlahakes ${ }^{1}$ \\ ${ }^{1}$ Massachusetts General Hospital
}

July 14, 2021

\begin{abstract}
There is no abstract, as this is an editorial comment.

To MUF or not to MUF, that is the question.

Jordan P. Bloom, MD, MPH

Cardiac Surgery Fellow, Massachusetts General Hospital

Gus J. Vlahakes, MD

Professor of Surgery, Harvard Medical School and Massachusetts General Hospital

Contact information for correspondence:
\end{abstract}

Gus J. Vlahakes, MD

Massachusetts General Hospital

55 Fruit Street, COX630

Boston, MA 02114

vlahakes.gus@mgh.harvard.edu

Phone: 617-726-1861

Professor Talwar and colleagues revisit the ultrafiltration debate by reporting a randomized prospective trial comparing conventional (CUF) vs. modified (MUF) ultrafiltration in pediatric patients undergoing operative repair of Tetralogy of Fallot (TOF) ${ }^{1}$. Of the 79 patients in the study, 40 were randomized to CUF only and 39 to CUF+MUF. The primary outcome was hematocrit, which was shown to be significantly higher in patients who received MUF (44.7 vs. 37.2, $\mathrm{p}<0.001$ ). The authors report numerous statistically significant differences in secondary outcomes, however the clinical significance of many of these is negligible (CVP, peak airway pressures, and inotrope score). Two of the secondary outcomes were clinically very different. Time to peripheral rewarming was 6.3 hours in the CUF+MUF group vs. 13.7 hours in the CUF only group. This finding shows a remarkable difference; the other significant finding was a difference in mechanical ventilation time: 14.7 hours vs. 6.3 hours. Finally, cardiac biomarkers and inflammatory markers were measured in the patients. Of these, there was a remarkable difference in troponin between groups with the CUF+MUF group being much lower (231 vs. 5057). The others have negligible clinical significance.

Modified ultrafiltration involves spending 20-30 minutes running the circuit and patient blood through a hemofilter after discontinuation of cardiopulmonary bypass. This technique was originally described by Naik et al in $1991^{2}$ and has been shown to reduce the total body water concentration ${ }^{3,4}$. As the authors accurately point out, there have been numerous studies reporting mixed results about the potential benefits 
of MUF. We think it is safe to say that other than additional time in the operating room on bypass, there are no clear disadvantages to ultrafiltration. The real question to ask about this study and the technique of MUF is whether it confers any improvement in outcomes? A metanalysis by Ziyaeifard et al. evaluated 65 papers published between 1987 and 2013 and concluded that there is an association between MUF and an attenuation in mortality after pediatric cardiac surgery ${ }^{5}$. They further conclude that MUF results in wellknown advantages in children with improvements in the hemodynamic, pulmonary, coagulation and other organs functions and results in decrease in blood transfusion, reduction of total body water, and blood loss after surgery. Unfortunately, all the data referenced in both the metanalysis and this manuscript are older than 10 years. Over the past decade there have been drastic changes in the way that perfusionists setup and pump pediatric patients. These include the use of blood priming, autologous priming and reduction in circuitry volume, all which result in hemoconcentration of the perfusate ${ }^{5,6}$. These advances question the need for MUF and it is not clear from this manuscript whether these are routine practices at the authors' institution. More studies are needed to compare outcomes in the modern era, and we applaud the authors for a timely study.

There are several issues with this trial. First, the authors report it is a double-blinded study. Indeed, there were blinded participants in the study however the authors were not blinded and thus they are subject to the typical bias of a non-blinded study. We feel this study should not be referred to as a double-blinded trial. Clearly the authors have redemonstrated what is already known, that there is an inverse relationship between time spent ultrafiltrating and total body water resulting in higher a hematocrit ${ }^{7}$. Interestingly, despite this there was no difference in rate of donor blood transfusion in either group. So, is more better? There is a dramatic reduction in time to re-warming and time to extubation in the CUF+MUF group. The authors state that patients are not considered for extubation until they have achieved peripheral rewarming - As such, these two variables are colinear and the difference in mechanical ventilation time is almost assuredly due to the difference in time to rewarming. One might postulate that a decrease in the time to achieving rewarming might result in less post-operative bleeding. Unfortunately, the patients who bled post-operatively were excluded from the study. This is curious and we think those patients should have been included in the analysis, particularly because there were more of them in the CUF only group. Another very interesting albeit statistically insignificant finding was the rate of ventilator associated pneumonia (VAP). Three patients died from VAP in the CUF only group compared to one patient in the CUF+MUF. Clearly this study was underpowered to detect differences in mortality after TOF repair in $20 \mathrm{~kg}$ children, which should be close to zero. That said, the authors might be on to something since we already know that time on the ventilator is predictive of VAP. With respect to the biomarkers, there are a number of studies that have looked at the relationship between troponin and hemofiltration. In this study, MUF must be clearing troponin from the bloodstream, thus accounting for the dramatic post-operative differences. It is not clear, nor addressed in this study, whether this finding has any clinical significance. Finally, the authors conclude that there were no significant between group differences in mortality, ICU or hospital length of stay.

As was pointed out accurately in the manuscript, there is mixed evidence on whether prolonging the ultrafiltration process after discontinuation of CPB confers any benefit to the patients. To summarize the literature at this point, it is safe to say that we don't know the answer to this question but despite small differences here and there, this trial failed to demonstrate any long-term benefit and likely will not change anyone's opinions about the addition of MUF to CUF.

\section{References:}

1.Talwar S, Sujith NS, Rajashekar P, Makhija N, Sreenivas V, Upadhyay AD, et al. Modified ultrafiltration \& postoperative course in patients undergoing repair of Tetralogy of Fallot. J Card Surg

2. Naik SK, Knight A, and Elliott, MJ. A successful modification of ultrafiltration for cardiopulmonary bypass in children. Perfusion 1991;6:41-50.

3. Shen J, Wang W, Zhang W, Jiang L, Yang YY. A high-efficiency MUF method benefits postoperative hemodynamic stability and oxygen delivery in neonates with transposition of the great arteries. J Card Surg 
2019;34:468-473.

4. Koutlas TC, Gaynor JW, Nicolson SC, Steven JM, Wernovsky G, and Spray TL. Modified ultrafiltration reduces postoperative morbidity after cavopulmonary connection. Ann Thorac Surg 1997;64:37-42; discussion p. 43.

5. Ziyaeifard M, Alizadehasl A, and Massoumi G. Modified ultrafiltration during cardiopulmonary bypass and postoperative course of pediatric cardiac surgery. Res Cardiovasc Med 2014;3:e17830.

6. Mejak BL, Lawson DS, and Ing RJ. Con: Modified ultrafiltration in pediatric cardiacsSurgery is no longer necessary. Journal of Cardiothoracic and Vascular Anesthesia 2019;33:870-872.

7. Harvey B, Shann KG, Fitzgerald D, Mejak B, Likosky DS, Puis L, et al. International pediatric perfusion practice: 2011 survey results. J Extra Corpor Technol 2012;44:186-193. 